\title{
A PKI-based mobile banking demonstrator
}

\author{
No Author Given \\ No Institute Given
}

\begin{abstract}
This paper presents a home banking solution for mobile phones, using a secure micro-SD card that features a smart card chip. This card is used to implement a strong online authentication with the bank server, based on a public key infrastructure, providing a flexible way to add entities - users as well as banks - to the ecosystem. The implemented system is running on Android mobile phones, taking into account the possible weaknesses at operating system level. The microSD card is running Java Card 2.2.1. Different security features are discussed that considerably improve upon existing mobile banking systems and allow for seamless integration of our system in the current smart phone context.
\end{abstract}

\section{Introduction}

Very soon after mobile phones were launched throughout the world, system developers thought of offering a banking application on users' cell phones that would allow them to manage their bank account any time, any place. The mobile phones not having the computer power they have today, these systems were quite rudimentary and their security flexibility was based on the SIM Toolkit which allows for secret key sharing between the bank and the phone's SIM card [8, 2]. As mobile phones today can be considered complete computing devices, more complex and thus useful mobile banking applications can be developed that can lead to larger user acceptance. This is facilitated by the growing number of smart phones and the increasing penetration of mobile Internet access. In Belgium, BNP Paribas Fortis started its mobile banking system in September 2010, after smaller banks like Keytrade rolled out this functionality already in 2009. the fact that a large bank like BNP Paribas is moving in this arena, indicates that mobile banking is becoming a common service in the banking world. This phenomena will only grow, and we expect it to be part of the standard daily banking package within a couple of years.

\section{Existing mobile banking products}

Nevertheless, the security of the available mobile banking systems remains debatable, as was also the case with Internet banking in its early days. The technology still has to mature, and some emerging solutions are therefore actually based on the Internet banking security solutions, involving separate security tokens, card 
readers etc. Although useful, mobile users need more compact and integrated solutions, in which the complete functionality is embedded in the mobile phone. However, it remains an open question whether or not the current solutions can be as secure as their non-mobile counterparts used in a more closed desktop environment. In this section we will briefly discuss two existing mobile banking systems while the next section will describe our own, fully integrated design.

In Belgium, at least two banks currently offer a mobile banking system: BNP Paribas Fortis [3] and Keytrade Bank [9]. Both banks provide a mobile banking site accessible via a smart phone's browser. The connection is secured by the HTTPS protocol. The authentication to the mobile banking server is a twofactor authentication, based on what the user knows, namely passwords/PINs and on what the user has, in this case a second communication or information channel (SMS, card reader, secure token). In Keytrade Mobile Banking, a RSA SecurID token [14] (what the user has) is combined with a password (what the user knows). This RSA secure token is a piece of hardware that generates an One-Time Password (OTP) at fixed time intervals (usually 30 or 60 seconds) using a built-in clock and a factory-encoded random key (known as the seed). The seed is different for each token, and is loaded into the corresponding RSA SecurID server as the tokens are purchased. The bank's server can then verify the OTP generated by the user's token by querying the correct RSA SecurID server entry. In Fortis Mobile Banking, a One-Time Password (OTP) is sent to the user by SMS during the authentication phase. This OTP, coupled to the mobile phone number, constitutes a first authentication factor (what the user has). As for the Keytrade application, the password of the user is the second factor used (what the user knows). The alternative in Fortis Mobile Banking, is using a card reader and the debit card of the user, the equivalence of the ordinary Internet banking.

The systems that are specifically addressed to mobile banking are vulnerable to phishing and man-in-the-middle attacks. Because of the fact that a standard browser is used, the user can be diverted to a fake man-in-the-middle website, using a phishing SMS or email. The OTP generated by the RSA secure token or the OTP, sent by SMS can also be recovered this way. Another disadvantage, in case secure tokens or card readers are used, is that the user must always carry around this device together with his phone. In case the OTP is sent by SMS, this might also involve additional costs for the user; for BNP Paribas Fortis Mobile Banking, one free SMS per day is offered, but additional OTP request come at premium SMS rates. Another potential risk in using SMS is the communication channel: not only does this require a trust relation with the Mobile Network Operator (MNO), but also depends on the security properties of the used mobile network. In Belgium, mobile phone communications are encrypted using the A5/1 standard. This standard presents several serious weaknesses [6]. In 2010, researchers Karsten Nohl and Sylvain Munaut gave a live demonstration of their GSM attack at the 27th annual Chaos Communication Congress in Berlin [4]. The whole process takes about 20 seconds, enabling phone conversations and SMS messages to be recorded and decrypted [7]. Finally, even the 
secure hardware tokens (RSA SecurID) seem to have their weaknesses. A recent hack of the backend systems of $\mathrm{RSA}^{1}$, apparently revealed enough information to attack one of their customers using SecurID, Lockheed Martin. Breaches like these, were the token's seed are revealed, bring back these system's security to just one authentication factor (the user's password), which has been shown to be rather insecure.

On the other hand, a fully integrated mobile system as presented in the next section obviously lacks the duality of two separate information sources or factors (namely the phone and some external token or channel) used in these existing applications. As will be explained in section 5, the proposed system therefor implements different security features to compensate this.

\section{System overview}

Building mobile banking application based on shared secrets always implies some major risks and is often based on trust in third parties (MNO, RSA, etc.). For this reason an alternative way to the existing mobile banking systems is proposed, based on a Public Key Infrastructure (PKI), built around what is called a secure element (SE) inside the mobile phone. Another obvious advantage of this approach is the absence of additional devices and tokens such as a card reader or a secure token.

The main feature of an SE is that it is considered secure against known hardware and software attacks. It typically consist of secure memory, a processor and one or more cryptographic co-processors. These characteristics allow developers to use SEs for storing cryptographic keys or other critical data and to securely deploy cryptographic functions in their applications.

Different types of SEs exist, although most of them today are so-called Java Cards [12]. These Java Cards are SEs on which a very limited subset of the Java Virtual Machine (JVM) is running, with a reasonable range of security functions available. Another aspect to SEs is that they come in different form factors. The SE can either be embedded into the phone, stored inside a removable microSD card or sit on the phone's SIM card. In our application we used a microSD card that supports Java Card version 2.2.1.

Secure microSD cards are produced by several manufacturers; we used a card developed by the German company Giesecke \& Devrient [5]. The card architecture is shown in Figure 1. A smart card chip is contained inside the micro-SD card. One accesses it via the flash controller which acts as a proxy, similar to a smart card reader. The APDUs, used to address the smart card are tunnelled via specific SD read/write commands. Towards the developer the smart card is presented as a classic Java Card, allowing a quick take-up and easy portability of the developed application to other types of SEs.

The banking application has been developed as a stand-alone application on a smart phone running the open Android OS containing this microSD card. The

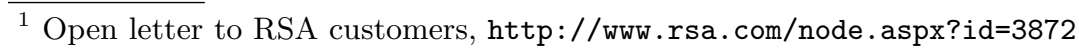




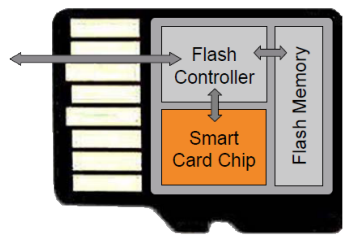

Fig. 1. Mobile Security Card Architecture

bank server has been simulated by a pseudo banking application running on Apache Tomcat [15], using Java Servlet technology. A comprehensive overview of our solutions, including a symmetric key version of our application, can be found in [10].

Summarised, the components in the system, depicted in Fig 2, are the following:

- The secure microSD card (further referred to as 'the card'): this is used as the root of trust at the user's side. It holds cryptographic key material that never leaves the card. The Java Card program on the card, is referred to as the applet.

- The mobile phone, with a dedicated application for mobile banking. The application implements the communication gateway between the secure microSD card and the mobile banking server. It also provides the GUI to the user.

- The mobile bank server, providing the interface to the bank's back-end systems.

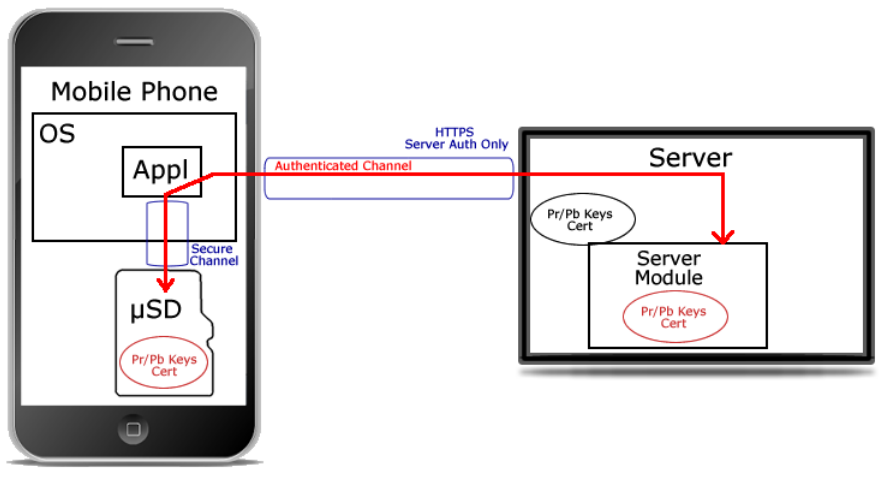

Fig. 2. Diagram of the proposed mobile banking setup 


\section{The mobile banking application}

Our solution solution features a small Public Key Infrastructure (PKI). Both the card and the server have their own X.509 public certificate and associated private key, as shown in Figure 3. The card's certificate and private key are stored inside the card's applet. This private key is actually generated inside the card at the card's personalisation stage, preventing the private key to ever leave the card, solving issues previously mentioned regarding shared secrets. The X.509 certificates, used in the communication with the mobile banking server, are parsed and analysed directly in the smart card. The reason for this is that we have designed the applet in the card to be independent of the bank. This way, we can decouple the issuing of the card and its private key from an individual bank. While it remains possible for a bank to deploy and manage its own secure microSD card, such a secure element is typically governed by a independent third party, such as a mobile network operator or a Trusted Service Manager (TSM, e.g. Venyon). Our generic card applet therefore allows for the bank to focus on the android application for branding and differentiation, while the trusted third party takes care of the secure applet deployment. This way, the complex ecosystem of deploying secure applets on mobile phones [1] gets simplified and customers can use the same applet for different mobile banking applications on their phone.

Unfortunately, the dependence on PKI certificates presented a complication: there is no Java class in the Java Card 2.2.1 specification to handle X.509 certificates. The existing proprietary implementations were not freely available to us, so we implemented our own X.509 certificate parser.

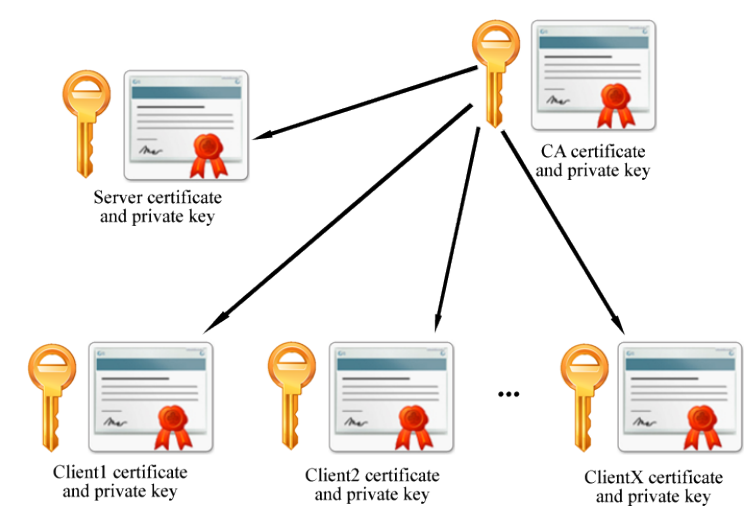

Fig. 3. PKI structure of the implementation

In what follows, we describes our application in more detail. After presenting the general authentication and key establishment methods used, more specific security features are described, protecting against certain dedicated attacks. A 
last subsection gives an overview of a mobile banking transaction as it is executed within our application.

\subsection{Authentication and key establishment}

The protocol, used for mutual authentication and (symmetric) key agreement between the card and the mobile banking server, is based on the "X.509 strong three-way authentication" protocol [11]. In our protocol, the micro-SD card and the server exchange X.509 certificates and signatures in order to prove their identity. The symmetric session keys are generated by the bank server and sent to the card, encrypted by the card's public key. The session keys are then used for the subsequent communication. One major advantage of asymmetric mobile banking is that the server must not store the client's keys anymore. Furthermore, this private key now resides in the user's hardware (card) only. Another advantage is the facility of deploying the system. The distribution of the public key certificates can be done independent of the server setup. As already mentioned, the cards generate their own keypair, and send a Certificate Signing Request (CSR) to the applet's manager (e.g., a TSM like Venyon), using a dedicated, bank-independent application. Upon successful verification of such a CSR, the TSM will issue and send a certificate for the card's public key that will be loaded into the applet.

Below, we describe the protocol's details.

\section{Notation}

SUMMARY: The card $A$ and server $B$ exchange 4 messages, after the user $I$ has introduced his PIN code to confirm his authenticity to the card.

RESULT: Mutual entity authentication and session keys transport with key authentication.

- $P_{X}(y)$ denotes the result of applying $X$ 's encryption public key to data $y$.

- $S_{X}(y)$ denotes the result of applying $X$ 's signature private key to data $y$.

$-r_{A}, r_{B}$ are freshly generated random numbers (to detect replay and impersonation).

- cert $_{X}$ is a certificate, binding party $X$ to a public key suitable for both encryption and signature verification.

\section{System Setup}

(a) Each party has its public key pair for signing and encryption.

(b) $A$ must acquire (and authenticate) the public key of $B$ a priori.

\section{Protocol messages}

$$
\begin{gathered}
A \leftarrow I: P I N \\
A \rightarrow B(\text { via I }): B, \operatorname{cert}_{A}, r_{A}
\end{gathered}
$$




$$
\begin{aligned}
& A \leftarrow B(\text { via I }): A, \operatorname{cert}_{B}, r_{B} \\
& A \rightarrow B(\text { via I }): S_{A}\left(r_{A}, r_{B}, B\right) \\
& A \leftarrow B(\text { via I }): P_{A}\left(W_{1}, W_{2}\right), S_{B}\left(r_{A}, r_{B}, A, P_{A}\left(W_{1}, W_{2}\right)\right)
\end{aligned}
$$

\section{Protocol actions}

- The user enters his PIN code. Upon successful verification by the card, the card is unlocked. $A$ sends $B$ 's identifier (for example the subject common name of $\operatorname{cert}_{B}$ : www.nameofthebank.com), its own certificate $\operatorname{cert}_{A}$ and a fresh random number $r_{A}$.

- The server verifies that the identifier in the previous message is correct. It verifies the certificate $\operatorname{cert}_{A}$ and stores the challenge $r_{A}$. It then sends the identifier of the client $A$ (which can also be the common name of $\operatorname{cert}_{A}$ ) together with its own certificate $\operatorname{cert}_{B}$ and a new challenge $r_{B}$.

- The card verifies $B$ 's certificate $\operatorname{cert}_{B}$, verifies that the identifier is correct and stores the challenge $r_{B}$. Upon successful verification, the card sends a signature of both challenges and $B$ 's identifier using the client's private key.

- The server $B$ verifies the signature using $A$ 's public key, thereby verifying that $r_{A}, r_{B}$ and $B$ (transmitted earlier in the previous messages) are indeed consistent.

- The server then randomly generates a session key $W_{1}$ and a MAC key $W_{2}$ that will be used as symmetric keys for subsequent communications. These keys are encrypted with $A$ 's public key. The server also sends a signature of $r_{A}, r_{B}, B$ and $P_{A}\left(W_{1}, W_{2}\right)$ using its private key.

- $A$ decrypts the session key $W_{1}$ and the MAC key $W_{2}$ using its private key, and verifies the signature using $B$ 's public key.

\section{Limitations}

One major limitation of this setup is the processing power of the secure microSD card. The authentication time is about 6 seconds with the material used, and this is mainly due to the signature generation and verification and the session key decryption in the card.

\subsection{Two-layered security}

On top of the previously discussed key establishment between the card and the backend server our implementation makes use of an TLS/SSL connection between the phone's application and the backend. The reason we added this extra layer of security is twofold. The first reason is to ensure data confidentiality and integrity already at the application level. This way, basic application level data transfer and authentication can be specifically implemented according to the bank's policy and vision. It allows for direct backend to user communication as shown in Figure 2, without passing by the slower secure card. This connection should nevertheless only be used for less critical data exchange, especially as 
the SSL protocol is executed without strong client authentication. In our implementation this is for example used for retrieving the user's account balance and history, but does not allow for setting up a money transfer. The other reason for this second layer of security is to prevent the user's personal applet certificate used during the applet level authentication protocol and other transaction information to be sent in the clear to the backend, thereby increasing the user's privacy.

The user authentication and key establishment described in the previous section should be used for the most critical transactions and is independent of the TLS/SSL tunnel through which it runs. This way, we obtain a two layered security architecture that allows for a robust mobile banking application that provides for confidentiality, integrity, secure client authentication and secure client authorisation.

In our implementation, the microSD card (and hence two layers of security) is used when initially authenticating to the mobile banking server, and for every money transfer. Of course, it is ultimately up to the banks to decide which features to put at which layer.

\subsection{Securing the OS to card communication}

As can be seen in Figure 2, another authenticated channel is set up inside the mobile phone, between the application running inside the Android operating system and the applet contained in the secure element. This is necessary because the Android banking application has now become the weakest link in the chain: it has to run in the Android OS, which can be compromised easily, as recent research has shown ${ }^{2}$ or can at least easily be fooled to let users install malicious applications. The reason we thus need to set up yet another secure channel between the genuine application and the secure card is to prevent these malicious applications or code but also other unauthorised users or the user himself to abuse the secure card's critical applets. These applets are usually very sensitive and often protected by a limited number of PIN tries. Abusing these PIN tries can block the applet and therefor allows for an easily implemented denial of service attack.

Our implementation of such a secure channel as described by the Global Platform consortium [13] prevents that any external application can communicate with the card's applet, even when the PIN code is known. Secondly, it ensures that attackers with eavesdropping or modification abilities on the communication channel between the application and the applet can't influence the system's security.

During the installation of the applet on the secure microSD card, the client will enter a activation code, to be used in the mobile banking card applet installation application (at OS level). Using this application code, a long-term symmetric key is set up between the banking application and the applet in the

\footnotetext{
${ }^{2}$ The Register - Toxic Plankton feeds on Android Market for two months; http: //www.theregister.co.uk/2011/06/13/android_market_still_insecure/
} 
card. This allows the application to authenticate to the applet later on, each time setting up a new encryption key $S_{1}$ and MAC key $S_{2}$ for the encrypted tunnel between application and applet. Note that this still allows for some attacks if the long-term symmetric key is recovered by an attacker. Securing this longterm key can be done using several existing (but not implemented) technologies, including:

- Obfuscation and steganography: the long-term key can be hidden in memory using classical obfuscation and steganographic techniques. In storage, a password-based encryption, combined with steganography and the classic access control features of Android, will be the best we can do.

- Another possibility, is the use of so-called white-box cryptography, in which the long-term symmetric key is hidden in the implementation of the application. The authors have not seen white-box cryptography in mobile phones at the time of writing, but it appears that the technology is ready to be used in this setting.

\subsection{Avoiding automated attacks}

Finally, to further avoid attacks on the OS level, a captcha ${ }^{3}$ system was implemented. Each time the user would make a transfer, a captcha is presented, that represents a simple human-only-readable challenge, depending on the data in the transaction.

Our captcha is therefore not a classic captcha with random letters but is adapted to the mobile banking system, as shown in Fig 4(b). The idea is that the user receives, after each transfer, a summary of his transfer in the form of an image. Furthermore, a part of the image contains random letters generated by the bank server, the amount of the transaction inserted randomly among the letters and finally a box in which the user must make a calculation, based on digits issued from the recipient's account number. This way, the user can also verify his transaction data one more time before confirming it.

Involving such a captcha ensures that a human must be present during a transaction. For each transaction, an attacker will need to get involved to solve the captcha, and this complicates the design of an automated and distributed attack. Moreover, injecting/replacing other beneficiary account numbers in a transaction in construction, and having this signed by the user is harder: the response of the server will show the wrong captcha and the user can be watchful for abnormal behaviour of his application..

\subsection{Overview of a money transfer}

For actual money transfers, the secure microSD card is used to authenticate the transaction. The protocol is shown in Figure 5. The money transfer including the

\footnotetext{
${ }^{3}$ captcha - completely automated public Turing-test to tell computers and humans apart, http://www. captcha.net
} 


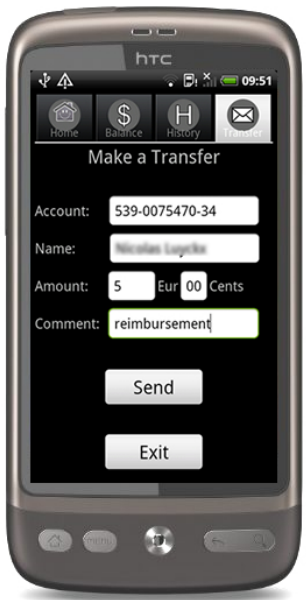

(a) Make a transfer

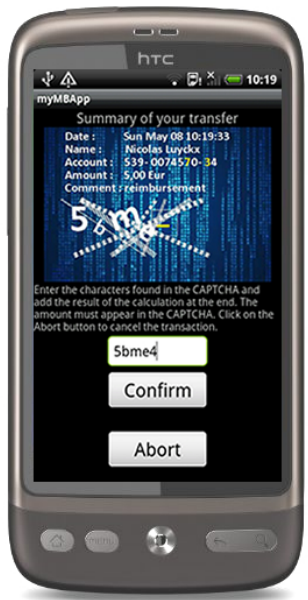

(b) Resolve the captcha

Fig. 4. Screen captures of the implemented Android application

amount and the recipient's account number is first sent to the secure element via the authenticated channel using the session keys $S_{1}$ (encryption key) and $S_{2}$ (MAC key) coming from the unilateral authentication between the card and the application. The card decrypts the message and encrypts it again using the session keys $W_{1}$ (encryption key) and $W_{2}$ (MAC key) shared between the card and the bank server. The encrypted message is sent, over the application, through the SSL connection, to the server. The server then responds it has received the transfer. The application then connects again to the bank server and receives an image containing the summary of the transaction and a captcha. The user has to solve the captcha, forming a confirmation code. This code is sent to the card, in the same way as the original transfer. The result is then sent to the server which verifies the confirmation code. If it is not correct, the protocol is repeated a limited number of times.

\section{Conclusion and outlook}

In this paper, we proposed a PKI-based alternative way for existing mobile banking systems, using a secure micro-SD card inside a smart phone. It makes mobile banking truly mobile, without the need of additional hardware. The proposed application is less sensitive to attacks than other mobile banking solutions, that are typically using any browser that the mobile phone OS supports. The price for such an integrated system is that it cannot be switched off easily: the microSD card is continuously accessible for attacks, and a considerable amount of mobile phones is always online. However, this will be the case for all mobile banking implementations, especially if no additional, non-networked dedicated hardware is used. However, one big advantage of our system is that every money transfer 


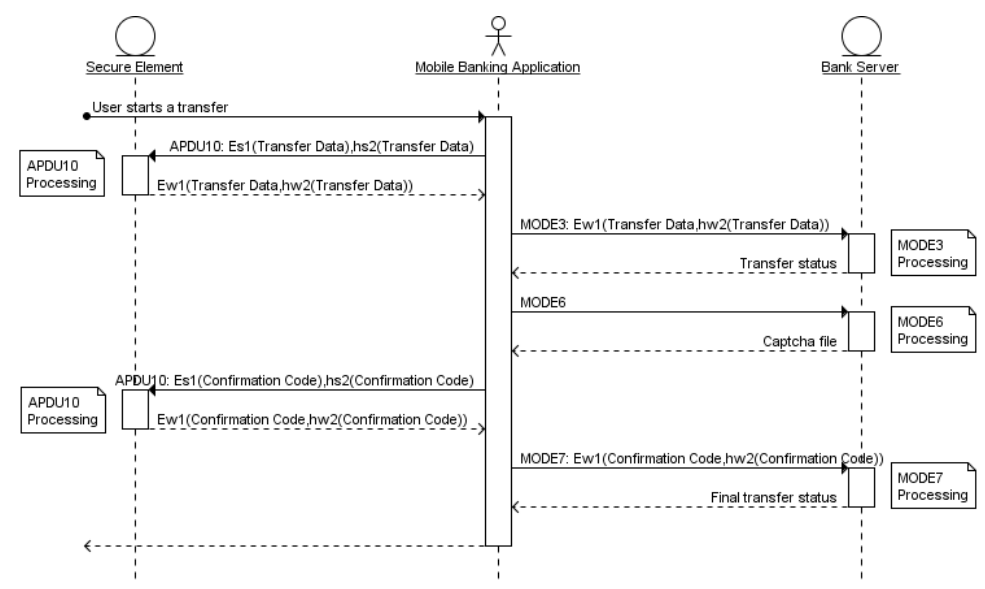

Fig. 5. UML sequence diagram for sending a transfer

will necessarily go through the secure microSD card. The necessary credentials to authorise these transfers cannot be extracted from the card without breaking its strong security features.

Future work includes the implementation of the protection mechanisms (obfuscation, steganography and white-box cryptography) for the long-term symmetric key that protects the link between application and applet. Another security improvement (but speed drawback) would be to include another public key pair dedicated to signing money transfers. Finally, as more and more mobile phones come without microSD card slot, it would be interesting to port this work to a suitable SIM card or other embedded secure elements as on the recent Google/Samsung Nexus S phone and to implement the banking application on other OSs as well.

\section{References}

[1] The Smart Card Alliance. Proximity Mobile Payments: Leveraging NFC and the Contactless Financial Payments Infrastructure. 2007. URL: http: //www. smartcardalliance.org/pages/publications-payments-mobil e-payments-nfc.

[2] Atos Worldline S.A./N.V. Banksys mobile banking application: m-banxafe. 2008. URL: http://www . atosworldline. be/landing-banxafe.html.

[3] BNP Paribas Fortis. 2011. URL: https://www.bnpparibasfortis.be.

[4] CCC. 27th Chaos Communication Congress. 2010. URL: http://events. ccc.de/congress/2010/wiki/Main\_Page.

[5] Giesecke \& Devrient. 2011. URL: http://www.gi-de.com/en/index.jsp. 
[6] Steve Gold. "Cracking GSM". In: Network Security 2011.4 (2011), pp. 12 -15. ISSN: 1353-4858. DOI: DOI : $10.1016 /$ S1353-4858(11) 70039-3. URL: http://www.sciencedirect.com/science/article/pii/S13534858117 00393.

[7] GSM Phones Now Vulnerable To Eavesdropping with Cheap Off-the-shelf Equipment. 2011. URL: http://www . livehacking.com/2011/01/04/gs m-phones-now-vulnerable-to-eavesdropping-with-cheap-off-theshelf-equipment/.

[8] S.B. Guthery and M.J. Cronin. Mobile application development with SMS and the SIM toolkit. McGraw-Hill telecom professional. McGraw-Hill, 2002. ISBN: 9780071375405.

[9] Keytrade Bank. 2011. URL: https://www.keytradebank.com.

[10] N L. "Secure Mobile Banking". MA thesis. (institute removed to protect anonymity of submission), 2011.

[11] Alfred J. Menezes, Scott A. Vanstone, and Paul C. Van Oorschot. Handbook of Applied Cryptography. 1st. Boca Raton, FL, USA: CRC Press, Inc., 1996. ISBN: 0849385237.

[12] Oracle. Java Card Technology. 2011. URL: http://www.oracle.com/tech network/java/javacard.

[13] Global Platform. Secure Channel Protocol 03. 2009. uRL: http: //www.g lobalplatform.org/specifications/card/GPC \_2\\%202\_D-Secure ChannelProtocol03-2nd-public \_review.pdf.

[14] RSA. RSA secureID. 2011. URL: http://www.rsa.com/node.aspx?id=11 56.

[15] The Apache Software Foundation. Apache Tomcat. 2011. URL: http://to mcat . apache.org/index.html. 